Research Article

\title{
The Prediction and Prognostic Significance of INPP5K Expression in Patients with Liver Cancer
}

\author{
Ruobing Wang $\mathbb{D}^{1},{ }^{1}$ Yan Jiao $\mathbb{D}^{1}$, Yanqing Li $\mathbb{D}^{2},{ }^{2}$ Siyang Ye, ${ }^{3}$ Guoqiang Pan, ${ }^{4}$ Shanshan Qin, ${ }^{5}$ \\ Fang Hua $\mathbb{D}^{6}{ }^{6}$ and Yahui Liu $\mathbb{D}^{1}$ \\ ${ }^{1}$ Department of Hepatobiliary and Pancreatic Surgery, The First Hospital of Jilin University, Changchun, Jilin 130021, China \\ ${ }^{2}$ Department of Pathophysiology, College of Basic Medical Sciences, Jilin University, Changchun, Jilin 130021, China \\ ${ }^{3}$ Department of Cardiology, The Second Hospital of Jilin University, Changchun, Jilin 130022, China \\ ${ }^{4}$ Department of Gastrointestinal Surgery, The First Hospital of Jilin University, Changchun, Jilin 130021, China \\ ${ }^{5}$ Department of Radiology, Affiliated Hospital of Qingdao University, Qingdao, Shandong 266000, China \\ ${ }^{6}$ Cardiovascular Internal Medicine, The First Hospital of Jilin University, Changchun, Jilin 130021, China
}

Correspondence should be addressed to Fang Hua; huafang360@163.com and Yahui Liu; liuyahui2008@yeah.net

Received 1 October 2019; Revised 4 March 2020; Accepted 13 March 2020; Published 27 April 2020

Academic Editor: Ian R. Ellis

Copyright (C) 2020 Ruobing Wang et al. This is an open access article distributed under the Creative Commons Attribution License, which permits unrestricted use, distribution, and reproduction in any medium, provided the original work is properly cited.

\begin{abstract}
Liver cancer is a devastating disease for humans with poor prognosis. Although the survival rate of patients with liver cancer has improved in the past decades, the recurrence and metastasis of liver cancer are still obstacles for us. Inositol polyphosphate-5phosphatase K (INPP5K) belongs to the family of phosphoinositide 5-phosphatases (PI 5-phosphatases), which have been reported to be associated with cell migration, polarity, adhesion, and cell invasion, especially in cancers. However, there have been few studies on the correlation of INPP5K and liver cancer. In this study, we explored the prognostic significance of INPP5K in liver cancer through bioinformatics analysis of data collected from The Cancer Genome Atlas (TCGA) database. Chi-square and Fisher exact tests were used to evaluate the relationship between INPP5K expression and clinical characteristics. Our results showed that low INPP5K expression was correlated with poor outcomes in liver cancer patients. Univariate and multivariate Cox analyses demonstrated that low INPP5K mRNA expression played a significant role in shortening overall survival (OS) and relapse-free survival (RFS), which might serve as the useful biomarker and prognostic factor for liver cancer. In conclusion, low INPP5K mRNA expression is an independent risk factor for poor prognosis in liver cancer.
\end{abstract}

\section{Introduction}

Hepatocellular carcinoma (HCC) is a devastating illness for humans, which has a poor prognosis with a relatively low 5 -year survival rate [1]. Despite therapies for patients with HCC are improving now, the recurrence and metastasis of liver cancer are still unsurmountable obstacles for us. Moreover, it is a great challenge to predict the clinical outcomes for HCC patients. Thus, it is significant to find an effective screening strategy such as new specific markers to identify prognosis for patients.

INPP5K belongs to the family of PI 5- phosphatase, which catalyze the dephosphorylation of the phosphate of the inositol ring on position 5 [2, 3]. It is also known as skeletal muscle and kidney-enriched inositol phosphatase (SKIP) [4]. INPP5K regulates the actin cytoskeleton, myoblast differentiation and insulin signaling in skeletal muscle [4-6]. Homozygous deletion of INPP5K in mice results in embryonic lethality [6]. Literatures have reported that mutations in INPP5K were associated with congenital muscular dystrophies, cataract, and intellectual disability $[5,7]$. Recently, PI 5- phosphatase has been reported to be associated with cell migration, polarity, adhesion, and cell invasion, especially in cancer cells. Moreover, the depletion of INPP5K may affect cell migration by the abundance of phosphatidylinositol 4,5bisphosphate $(\mathrm{PI}(4,5) \mathrm{P} 2)$ under the stimulation of integrin 
TABLe 1: Clinical characteristics of the liver cancer patients.

\begin{tabular}{|c|c|}
\hline Characteristics & Numbers of cases (\%) \\
\hline \multicolumn{2}{|l|}{ Age } \\
\hline$<55$ & $117(31.45)$ \\
\hline$\geq 55$ & $255(68.55)$ \\
\hline \multicolumn{2}{|l|}{ Gender } \\
\hline Female & $121(32.44)$ \\
\hline Male & $252(67.56)$ \\
\hline \multicolumn{2}{|l|}{ Histological type } \\
\hline Fibrolamellar carcinoma & $3(0.8)$ \\
\hline Hepatocellular carcinoma & $363(97.32)$ \\
\hline Hepatocholangiocarcinoma (mixed) & $7(1.88)$ \\
\hline \multicolumn{2}{|l|}{ Histologic grade } \\
\hline NA & $5(1.34)$ \\
\hline G1 & $55(14.75)$ \\
\hline G2 & $178(47.72)$ \\
\hline G3 & $123(32.98)$ \\
\hline G4 & $12(3.22)$ \\
\hline \multicolumn{2}{|l|}{ Stage } \\
\hline NA & $24(6.43)$ \\
\hline I & $172(46.11)$ \\
\hline II & $87(23.32)$ \\
\hline III & $85(22.79)$ \\
\hline IV & $5(1.34)$ \\
\hline \multicolumn{2}{|l|}{$\mathrm{T}$ classification } \\
\hline NA & $2(0.54)$ \\
\hline $\mathrm{T} 1$ & $182(48.79)$ \\
\hline $\mathrm{T} 2$ & $95(25.47)$ \\
\hline T3 & $80(21.45)$ \\
\hline $\mathrm{T} 4$ & $13(3.49)$ \\
\hline $\mathrm{TX}$ & $1(0.27)$ \\
\hline \multicolumn{2}{|l|}{$\mathrm{N}$ classification } \\
\hline NA & $1(0.27)$ \\
\hline No & $253(67.83)$ \\
\hline N1 & $4(1.07)$ \\
\hline $\mathrm{NX}$ & $115(30.83)$ \\
\hline \multicolumn{2}{|l|}{ M classification } \\
\hline M0 & $267(71.58)$ \\
\hline M1 & $4(1.07)$ \\
\hline MX & $102(27.35)$ \\
\hline \multicolumn{2}{|l|}{ Radiation therapy } \\
\hline NA & $25(6.7)$ \\
\hline No & $340(91.15)$ \\
\hline Yes & $8(2.14)$ \\
\hline \multicolumn{2}{|l|}{ Residual tumor } \\
\hline NA & $7(1.88)$ \\
\hline R0 & $326(87.4)$ \\
\hline $\mathrm{R} 1$ & $17(4.56)$ \\
\hline $\mathrm{R} 2$ & $1(0.27)$ \\
\hline $\mathrm{RX}$ & $22(5.9)$ \\
\hline
\end{tabular}

TABle 1: Continued.

\begin{tabular}{lc}
\hline Characteristics & Numbers of cases (\%) \\
\hline Vital status & $130(34.85)$ \\
Deceased & $243(65.15)$ \\
Living & \\
Sample type & $371(99.46)$ \\
$\quad$ Primary tumor & $2(0.54)$ \\
$\quad$ Recurrent tumor & \\
Os_s & $237(64.58)$ \\
0 & $130(35.42)$ \\
1 & \\
Rf_s & $179(55.94)$ \\
0 & $141(44.06)$ \\
1 & \\
INPP5K & $225(60.32)$ \\
High & $148(39.68)$ \\
Low & \\
Type & $373(100)$ \\
1 &
\end{tabular}

[8]. The above research suggests the role of INPP5K in regulating the motility of cells, which is consistent with the characteristics of tumor metastasis and invasion.

However, the prognostic significance of INPP5K expression in liver cancer has not been reported yet. In this study, we intend to assess the independent prognostic value of INPP5K expression for overall survival of liver cancer patients. INPP5K expression in liver cancer was obtained from The Cancer Genome Atlas Liver Hepatocellular Carcinoma (TCGA-LIHC). Patients were divided into high and low INPP5K expression groups to explore the correlations between INPP5K expression and different clinical features of liver cancer. Our results indicated that INPP5K might be a biomarker for the diagnosis and prognosis of liver cancer.

\section{Materials and Methods}

2.1. Data Mining of TCGA Database. We collected the RNAsequencing expression results (level three) from The Cancer Genome Atlas (TCGA) dataset by using R software (version 3.6.1) [9]. The pathological and clinical information of these patients was gathered following their ID in TCGA dataset. The patients' information included their ages, genders, histological grades, clinical stages, and T/N/M classifications. The INPP5K mRNA expression was evaluated as $\log 2(x+1)$ scores, which were converted to normalized RNA-Seq by Expectation-Maximization (RSEM) values for the further analysis.

2.2. Data Mining of ICGC Data Portal and GSE14520 Database. The gene expression results and clinical information of patients were also gathered from the International Cancer Genome Consortium (ICGC, https://icgc.org/) and Gene Expression Omnibus (GEO, https://www.ncbi.nlm.nih .gov/geo/) dataset. The GSE14520 microarray expression 

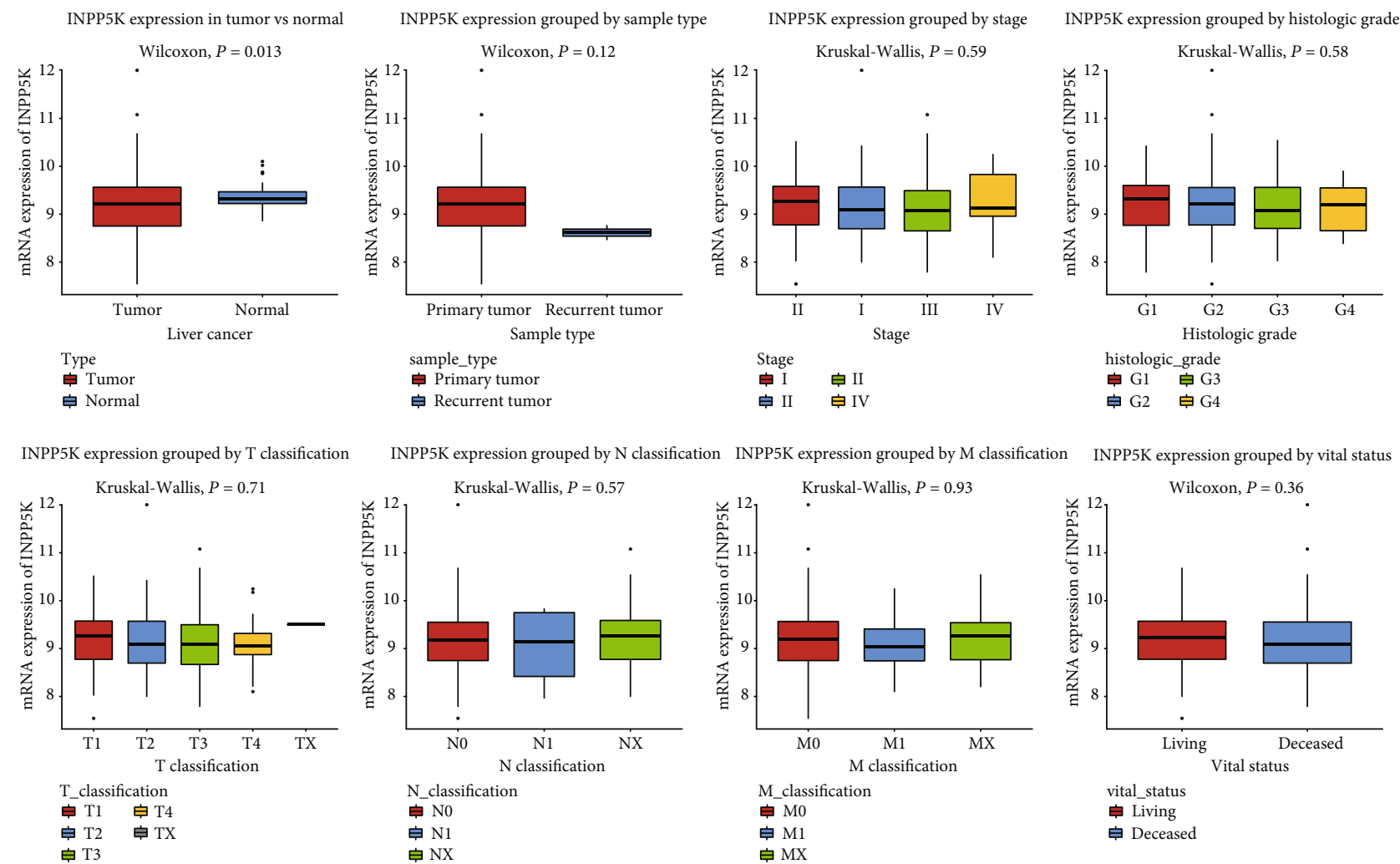

FIgURE 1: INPP5K expression in liver cancer. INPP5K expression of liver cancer tissues was compared with that in normal according to age, gender, histological type, histologic grade, TNM classification, radiation therapy, residual tumor status, vital status, sample type, overall survival, and relapse-free survival.

information was collected from GEO dataset. The results from TCGA dataset were verified by ICGC and GSE14520 dataset. These data were analyzed by $\mathrm{R}$ software.

2.3. Statistical Analyses. The expression of INPP5K in the TCGA-Liver Hepatocellular Carcinoma (LIHC) dataset was assessed using nonparametric rank sum tests and visualized in box plots. The Wilcoxon rank sum test was performed to analyze the differences between two subgroups, including the gender, age, and vital status. The Kruskal-Wallis test was performed to analyze the differences in three or more subgroups, including the histologic grades, clinical stages, and T/N/M classifications. To explore the relationship between INPP5K mRNA expression and clinical information, we drew the receiver operating characteristic (ROC) curve by using the pROC package [10]. According to the AUC value identified from the ROC curve, the samples were divided into high/low INPP5K mRNA expression groups. The correlation between clinical parameters and high/low INPP5K mRNA expression was conducted by Chi-square tests and Fisher exact tests. Kaplan-Meier analysis was performed to compare the differences in OS and RFS between high/low INPP5K mRNA expression groups based on the log-rank test using the survival package in $\mathrm{R}[11,12]$. Univariate Cox regression analysis was performed to select the potential prognostic factors by calculating the hazard ratios (HRs) and 95 percent confidence intervals (CIs). Multivariate Cox regression analysis was used to verify the correlation of
INPP5K mRNA expression with OS and RFS, along with the clinical parameters which were associated with clinical prognosis in univariate analysis. Statistical analysis was conducted with the $\mathrm{R}$ software (version 3.6.1). ${ }^{*} P<0.05$ was considered as significant.

\section{Result}

3.1. Patient Characteristics. Clinical information of 373 patients with liver cancer was downloaded and organized from The Cancer Genome Atlas Liver Hepatocellular Carcinoma (TCGA-LIHC), including age, gender, histological type, histologic grade, TNM classification, radiation therapy, residual tumor status, vital status, sample type, overall survival, and relapse-free survival (Table 1).

3.2. Low INPP5K Expression and Its Diagnostic Value in Liver Cancer. We compared the expression of INPP5K mRNA in liver cancer $(n=373)$ and normal liver $(n=50)$ tissue via box plots (Figure 1). The results indicated that INPP5K expression was lower in liver cancer $(P=0.013)$. Consistent with the results in the TCGA cohort, the mRNA expression of INPP5K was significantly downregulated in liver cancer tissues $(n=225)$ when compared with normal liver tissues $(n=220)$ in GSE14520 cohort $(P \leq 0.0001$; Supplementary Fig. S1).

The ROC curve was performed using the expression data from TCGA-LIHC to evaluate the diagnostic value of 

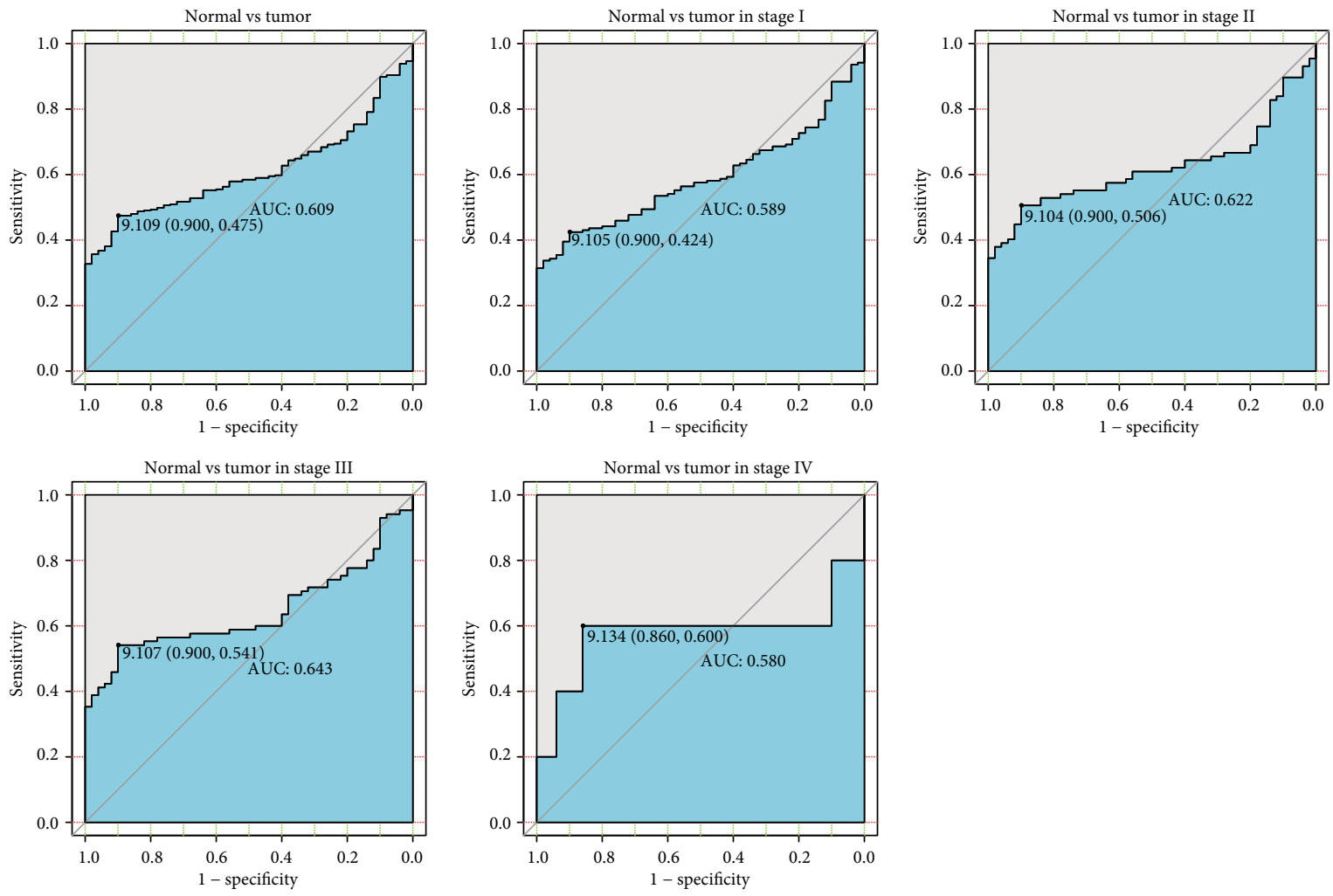

FIGURE 2: The diagnosis value of INPP5K mRNA expression in liver cancer. The ROC curve for INPP5K expression of liver cancer tissues was compared with that in normal. Subgroup analysis for stages I, II, III, and IV liver cancer.

INPP5K (Figure 2(a)). The area under the ROC curve (AUC) was 0.609 , which showed the modest diagnostic value of INPP5K. Subgroup analysis manifested the diagnostic value in different stages of liver cancer with the AUC values of $0.589,0.622,0.643$, and 0.580 for stage I, stage II, stage III, and stage IV, respectively (Figures 2(b)-2(e)).

\subsection{Correlations between Clinical Features and INPP5K} Expression in Liver Cancer. To assess the correlations between INPP5K expression and clinical features of liver cancer, patients were divided into high and low INPP5K mRNA expression groups according to the threshold determined by ROC curve (Table 2). The Chi-square test showed that low INPP5K expression was significantly correlated with survival status $(P=0.0276)$, overall survival $(P=0.0236)$, and relapse-free survival $(P=0.0004)$.

3.4. Low INPP5K Expression Is an Independent Risk Factor for Overall Survival in Liver Cancer Patients. To evaluate the diagnostic value of INPP5K in liver cancer patients, Kaplan-Meier survival curve with the log-rank test was executed, which indicated that low INPP5K expression was associated with poor overall survival $(P=0.0071$; Figure 3$)$. Subgroup analysis showed that low INPP5K expression was correlated with poor overall survival of cases with clinical stage II $(P=0.045$; Figure 3$)$. Consistent with the results in the TCGA cohort, the validation of survival analysis was conducted by ICGC cohort ( $P=0.025$; Supplementary Fig. S2).

Both univariate and multivariate Cox analyses showed that the expressions of INPP5K (hazard ratio $=1.5,95 \%$ confidence interval: $1.06-2.13, P=0.023)$, residual tumor (hazard ratio $=1.42,95 \%$ confidence interval: $1.11-1.82, P=$ 0.005 ), and $\mathrm{T}$ classification (hazard ratio $=1.81,95 \%$ confidence interval: 1.44-2.29, $P \leq 0.001$; Table 3 ) were independent risk factors for poor overall survival in liver cancer patients.

3.5. Low INPP5K Expression Is an Independent Risk Factor for Relapse-Free Survival in Liver Cancer Patients. To evaluate the diagnostic value of INPP5K in liver cancer patients, Kaplan-Meier survival curve with the log-rank test was executed, which indicated that low INPP5K expression was associated with relapse-free survival $(P \leq 0.0001$; Figure 4$)$. Subgroup analysis showed that low INPP5K expression was correlated with poor RFS of cases with clinical stage I/II $(P=0.0013$; Figure 4$)$ and stage III/IV $(P=0.039$; Figure 4$)$.

Univariate and multivariate Cox analyses showed that the expression of INPP5K (hazard ratio $=2.03$, 95\% confidence interval: $1.45-2.83, P \leq 0.001$ ), residual tumor (hazard ratio = $1.3,95 \%$ confidence interval: $1.02-1.66, P=0.035)$, and T classification (hazard ratio $=1.66,95 \%$ confidence interval: 1.27 2.15, $P \leq 0.001$; Table 4) were independent risk factors for RFS in liver cancer patients.

\section{Discussion}

Liver cancer is associated with a high mortality rate worldwide. Despite the rapid development of medicine, the recurrence and metastasis of liver cancer remain unsolvable. Prognostic markers have numerous potential roles in cancer. They can help to predict patients' outcomes to improve 
TABLE 2: Relationship between the clinical features of liver cancer and INPP5K expression.

\begin{tabular}{|c|c|c|c|c|c|c|c|c|c|}
\hline Parameter & Variables & $N$ & High & $\%$ & Low & $\%$ & $\mathrm{X} 2$ & $P$ & Fish \\
\hline \multirow{2}{*}{ Age } & $<55$ & 117 & 66 & $(29.46)$ & 51 & $(34.46)$ & 0.8127 & 0.3673 & 0.3615 \\
\hline & $\geq 55$ & 255 & 158 & $(70.54)$ & 97 & $(65.54)$ & & & \\
\hline \multirow{2}{*}{ Gender } & Female & 121 & 75 & $(33.33)$ & 46 & $(31.08)$ & 0.1166 & 0.7327 & 0.7346 \\
\hline & Male & 252 & 150 & $(66.67)$ & 102 & $(68.92)$ & & & \\
\hline \multirow{3}{*}{ Histological type } & Fibrolamellar carcinoma & 3 & 3 & $(1.33)$ & 0 & $(0)$ & 3.9605 & 0.138 & 0.1695 \\
\hline & Hepatocellular carcinoma & 363 & 216 & $(96)$ & 147 & $(99.32)$ & & & \\
\hline & Hepatocholangiocarcinoma (mixed) & 7 & 6 & $(2.67)$ & 1 & $(0.68)$ & & & \\
\hline \multirow{4}{*}{ Histologic grade } & $\mathrm{G} 1$ & 55 & 38 & $(17.19)$ & 17 & $(11.56)$ & 4.21 & 0.2397 & 0.2344 \\
\hline & G2 & 178 & 110 & $(49.77)$ & 68 & $(46.26)$ & & & \\
\hline & G3 & 123 & 66 & $(29.86)$ & 57 & $(38.78)$ & & & \\
\hline & G4 & 12 & 7 & $(3.17)$ & 5 & $(3.4)$ & & & \\
\hline \multirow{4}{*}{ Stage } & I & 172 & 109 & $(51.66)$ & 63 & $(45.65)$ & 1.2996 & 0.7292 & 0.7014 \\
\hline & II & 87 & 51 & $(24.17)$ & 36 & $(26.09)$ & & & \\
\hline & III & 85 & 48 & $(22.75)$ & 37 & $(26.81)$ & & & \\
\hline & IV & 5 & 3 & $(1.42)$ & 2 & $(1.45)$ & & & \\
\hline \multirow{5}{*}{$\mathrm{T}$ classification } & $\mathrm{T} 1$ & 182 & 115 & $(51.57)$ & 67 & $(45.27)$ & 2.2864 & 0.6832 & 0.7163 \\
\hline & $\mathrm{T} 2$ & 95 & 55 & $(24.66)$ & 40 & $(27.03)$ & & & \\
\hline & $\mathrm{T} 3$ & 80 & 45 & (20.18) & 35 & $(23.65)$ & & & \\
\hline & $\mathrm{T} 4$ & 13 & 7 & $(3.14)$ & 6 & $(4.05)$ & & & \\
\hline & $\mathrm{TX}$ & 1 & 1 & $(0.45)$ & 0 & $(0)$ & & & \\
\hline \multirow{3}{*}{$\mathrm{N}$ classification } & No & 253 & 150 & $(66.96)$ & 103 & $(69.59)$ & 0.5399 & 0.7634 & 0.6991 \\
\hline & N1 & 4 & 2 & $(0.89)$ & 2 & $(1.35)$ & & & \\
\hline & NX & 115 & 72 & $(32.14)$ & 43 & $(29.05)$ & & & \\
\hline \multirow{3}{*}{ M classification } & M0 & 267 & 159 & $(70.67)$ & 108 & $(72.97)$ & 0.4947 & 0.7809 & 0.7461 \\
\hline & M1 & 4 & 2 & $(0.89)$ & 2 & $(1.35)$ & & & \\
\hline & MX & 102 & 64 & $(28.44)$ & 38 & $(25.68)$ & & & \\
\hline \multirow{2}{*}{ Radiation therapy } & No & 340 & 208 & (97.65) & 132 & (97.78) & 0 & 1 & 1 \\
\hline & Yes & 8 & 5 & $(2.35)$ & 3 & $(2.22)$ & & & \\
\hline \multirow{4}{*}{ Residual tumor } & R0 & 326 & 198 & (90.41) & 128 & $(87.07)$ & 2.7596 & 0.4302 & 0.4237 \\
\hline & $\mathrm{R} 1$ & 17 & 8 & $(3.65)$ & 9 & $(6.12)$ & & & \\
\hline & $\mathrm{R} 2$ & 1 & 0 & $(0)$ & 1 & $(0.68)$ & & & \\
\hline & $\mathrm{RX}$ & 22 & 13 & $(5.94)$ & 9 & $(6.12)$ & & & \\
\hline \multirow{2}{*}{ Vital status } & Deceased & 130 & 68 & $(30.22)$ & 62 & $(41.89)$ & 4.8529 & 0.0276 & 0.0262 \\
\hline & Living & 243 & 157 & $(69.78)$ & 86 & $(58.11)$ & & & \\
\hline \multirow{2}{*}{ Sample type } & Primary tumor & 371 & 225 & $(100)$ & 146 & $(98.65)$ & 1.0481 & 0.3059 & 0.1568 \\
\hline & Recurrent tumor & 2 & 0 & $(0)$ & 2 & $(1.35)$ & & & \\
\hline \multirow{2}{*}{ Os_s } & 0 & 237 & 154 & $(69.37)$ & 83 & $(57.24)$ & 5.1222 & 0.0236 & 0.0193 \\
\hline & 1 & 130 & 68 & $(30.63)$ & 62 & $(42.76)$ & & & \\
\hline \multirow{2}{*}{ Rf_s } & 0 & 179 & 125 & $(64.1)$ & 54 & $(43.2)$ & 12.668 & 0.0004 & 0.0003 \\
\hline & 1 & 141 & 70 & $(35.9)$ & 71 & $(56.8)$ & & & \\
\hline
\end{tabular}

clinical decision-making and screen patients who are most likely to respond to the particular treatment. Therefore, it is important to find reliable biomarkers for diagnosis and prognosis in liver cancer. In recent years, bioinformatics has attracted much attention because of its significance in screening markers. We also have been working on the exploration of tumor biomarkers by bioinformatics [13-21].

In previous studies, some prognostic biomarkers of liver cancers have been identified through bioinformatics. The high mRNA expression of pescadillo (PES1), high mobility group A2 (HMGA2), microtubule-associated serine and threonine kinase 2 (MAST2), trophinin-associated protein (TROAP), and MEX3A was associated with poor prognosis for liver cancer [18, 22-25]. Among these biomarkers, MAST2, PES1, and HMGA2 are involved in the regulation of chromosomal instability, DNA replication, cell proliferation, and cell cycle progression. Meanwhile, TROAP and MEX3A are related to the adhesion and cell migration. On 


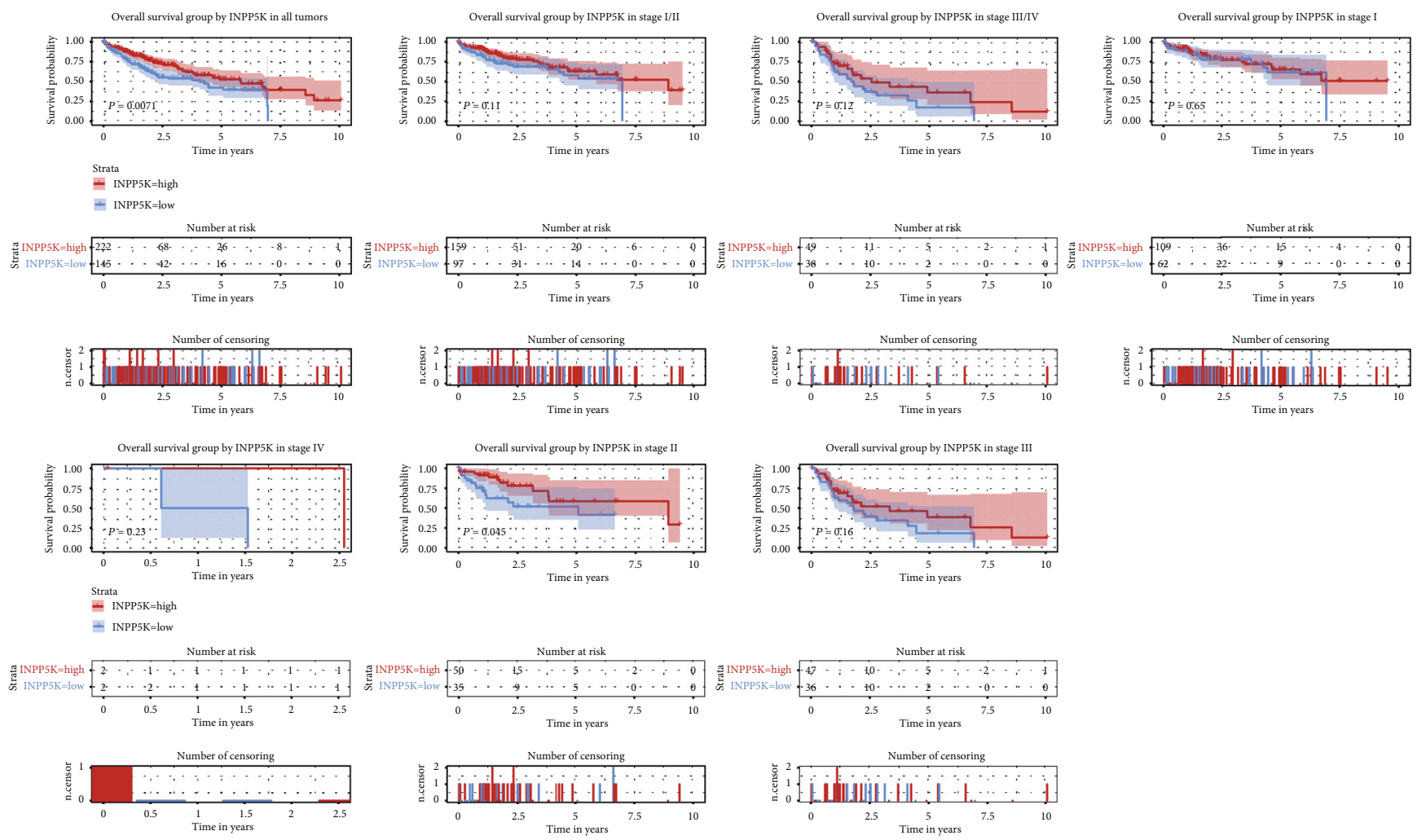

FIGURE 3: Kaplan-Meier curves for OS according to INPP5K expression in liver cancer. Survival analysis and subgroup analysis according to clinical stage were performed based on Kaplan-Meier curves.

TABLE 3: Univariate and multivariate analyses of the correlation of INPP5K expression with OS among liver cancer patients.

\begin{tabular}{|c|c|c|c|c|c|c|}
\hline \multirow{2}{*}{ Parameters } & \multicolumn{3}{|c|}{ Univariate analysis } & \multicolumn{3}{|c|}{ Multivariate analysis } \\
\hline & Hazard.Ratio.x & CI95.x & Pvalue.x & Hazard.Ratio.y & CI95.y & Pvalue.y \\
\hline Age & 1.00 & $0.69-1.45$ & 0.997 & & & \\
\hline Gender & 0.80 & $0.56-1.14$ & 0.220 & & & \\
\hline Histological type & 0.99 & $0.27-3.66$ & 0.986 & & & \\
\hline Histologic grade & 1.04 & $0.84-1.30$ & 0.698 & & & \\
\hline Stage & 1.38 & $1.15-1.66$ & 0.001 & 0.87 & $0.70-1.08$ & 0.205 \\
\hline T classification & 1.66 & $1.39-1.99$ & 0.001 & 1.81 & $1.44-2.29$ & 0.001 \\
\hline $\mathrm{N}$ classification & 0.73 & $0.51-1.05$ & 0.086 & & & \\
\hline M classification & 0.72 & $0.49-1.04$ & 0.077 & & & \\
\hline Radiation therapy & 0.51 & $0.26-1.03$ & 0.060 & & & \\
\hline Residual tumor & 1.42 & $1.13-1.80$ & 0.003 & 1.42 & $1.11-1.82$ & 0.005 \\
\hline INPP5K & 1.61 & $1.13-2.28$ & 0.008 & 1.50 & $1.06-2.13$ & 0.023 \\
\hline
\end{tabular}

the contrary, the low mRNA expression of oxoglutarate dehydrogenase-like (OGDHL) and phosphoglucomutaselike protein 5 (PGM5) was associated with poor prognosis for liver cancer $[16,26]$. These two biomarkers, acting as the putative tumor suppressor genes, play prominent roles in regulating the metabolic reprogramming process in cancers.

In this study, we found that INPP5K was lowly expressed in liver cancer and low expression of the INPP5K mRNA was associated with poor survival status and recurrence in liver cancer. Low INPP5K expression was correlated poor outcomes in liver cancer patients by the Chi-square test.
Univariate and multivariate Cox analyses demonstrated that INPP5K mRNA expression played a significant role in overall survival and relapse-free survival, which might be a useful biomarker and prognostic factor for liver cancer. The diagnostic value of INPP5K expression was also confirmed by Kaplan-Meier curves with the log-rank test for OS and RFS.

$\mathrm{PI}(4,5) \mathrm{P} 2$, a multifunctional lipid, is essential for regulating several basic subcellular processes in eukaryotic cells. It is also a key lipid messenger that regulates cell migration. Recently, the link between the dynamic balance of PI $(4,5) \mathrm{P} 2$ and the mechanisms driving cell polarity and migration has 


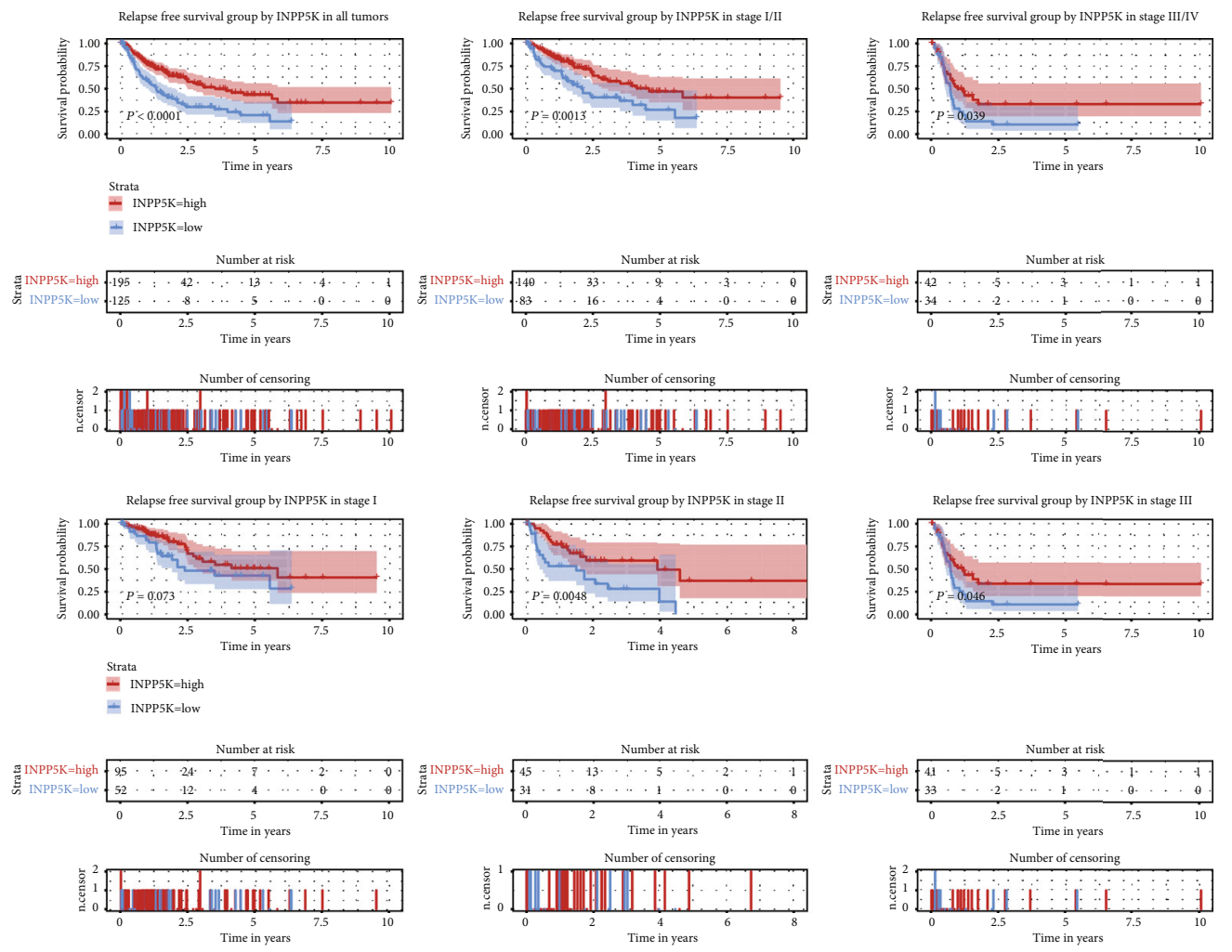

FIGURE 4: Kaplan-Meier curves for RFS according to INPP5K expression in liver cancer. Survival analysis and subgroup analysis according to clinical stage were performed based on Kaplan-Meier curves.

TABLE 4: Univariate and multivariate analyses of the correlation of INPP5K expression with RFS among liver cancer patients.

\begin{tabular}{|c|c|c|c|c|c|c|}
\hline \multirow{2}{*}{ Parameters } & \multicolumn{3}{|c|}{ Univariate analysis } & \multicolumn{3}{|c|}{ Multivariate analysis } \\
\hline & Hazard.Ratio.x & CI95.x & Pvalue.x & Hazard.Ratio.y & CI95.y & Pvalue.y \\
\hline Age & 0.90 & $0.63-1.28$ & 0.550 & & & \\
\hline Gender & 0.99 & $0.70-1.41$ & 0.966 & & & \\
\hline Histological type & 2.02 & $0.66-6.24$ & 0.220 & & & \\
\hline Histologic grade & 0.98 & $0.80-1.21$ & 0.883 & & & \\
\hline Stage & 1.66 & $1.38-1.99$ & 0.001 & 1.11 & $0.86-1.43$ & 0.416 \\
\hline $\mathrm{T}$ classification & 1.78 & $1.49-2.12$ & 0.001 & 1.66 & $1.27-2.15$ & 0.001 \\
\hline $\mathrm{N}$ classification & 0.97 & $0.67-1.40$ & 0.874 & & & \\
\hline M classification & 1.17 & $0.79-1.74$ & 0.432 & & & \\
\hline Radiation therapy & 0.74 & $0.26-2.16$ & 0.584 & & & \\
\hline Residual tumor & 1.28 & $1.01-1.61$ & 0.042 & 1.30 & $1.02-1.66$ & 0.035 \\
\hline INPP5K & 2.09 & $1.50-2.90$ & 0.001 & 2.03 & $1.45-2.83$ & 0.001 \\
\hline
\end{tabular}

been reported. It participates in the cytoskeletal organization via regulating related proteins (Mena, Tks5, or lamellipodin) [27]. Moreover, PI(4,5)P2 is involved in dynamic focal adhesion complexes and controls the migration and invasion of various cancer cells [28-30].

INPP5K is one of the PI 5-phosphatases, which catalyze the substrates such as $\mathrm{PI}(4,5) \mathrm{P} 2$ and $\mathrm{PI}(3,4) \mathrm{P} 2$ [2]. Similarly like the PI $(4,5) \mathrm{P} 2$, PI 5-phosphatases also have been reported to regulate cell migration and invasion in cancer cells. In glioblastoma, the decrease of SH2 domain-containing inositol 5phosphatase 2 (SHIP2) expression may have a positive or negative effect on cell migration rates depending on the types $[31,32]$. In addition, PI 5-phosphatase SHIP2 or INPP5K can be located on the plasma membrane and reduce the abundance of $\mathrm{PI}(3,4,5) \mathrm{P} 3$ or $\mathrm{PI}(4,5) \mathrm{P} 2$ [8]. Therefore, the association of low INPP5K expression with poor survival in liver cancer patients may be due to the effect of INPP5K on cell migration and invasion by controlling the abundance of $\mathrm{P}$ $\mathrm{I}(4,5) \mathrm{P} 2$. Besides, our results showed that low INPP5K expression correlated significantly with poor RFS in liver cancer cases of nearly all clinical stage except the stage I, which suggested that INPP5K might not regulate the migration at the 
initiation of tumor formation. As the expression of INPP5K was lower in the deceased than in the living, the relationship between INPP5K and survival needs to be further explored.

Gene expression and genetic characteristics of tumor are relevant to clinical features, pathological features, and the prognosis of the patients. Data from genomic profiling suggested there are two major molecular clusters (proliferation and nonproliferation) in liver cancer with differential enrichment in prognostic features and the activation of signaling pathway. In the proliferation signaling pathway, the expression of several biomarkers, namely, NOTCH, TGF- $\beta$ proteins, and several genes, is primarily associated with poor prognosis of patients [33]. It has been reported that the signature of 186 genes in liver cancer surrounding tissues can predict the higher risk of tumor recurrence after resection for liver cancer patients [34]. And these results are also associated with outcomes of patients with hepatitis C-related early-stage cirrhosis [35]. In addition, some clinical trials are being conducted based on the high MET expression and the mutations of RAS in tumor cells to find the potential biomarkers for predicting advanced HCC [36]. These studies suggest the feasibility of conducting tissue biomarker studies in patients with liver cancer. Therefore, the genetic biomarkers correlated with the prognosis of liver cancer should be more widely validated in clinical trials.

This is the first study that suggested the relationship between INPP5K and clinical characteristics in liver cancer patients by mining TCGA database so far, which indicated that low INPP5K mRNA expression may serve as an independent prognostic factor for poor survival in liver cancer. However, due to sample size limitation, it is difficult to establish a predicting model for INPP5K expression and clinicopathological variables in liver cancer. In further study, we will expand the sample size to explore the prognostic value of INPP5K expression and build an appropriate predicting model for the prognosis of liver cancer patients.

\section{Conclusions}

In this study, we assessed the independent prognostic value of INPP5K expression by mining TCGA database. Our results demonstrated that INPP5K was lowly expressed in liver cancer. The decreased expression level of INPP5K was related to poor prognosis, which could act as an independent risk factor for OS and RFS in liver cancer patients. This finding identified that low INPP5K expression was an independent factor involved in the prognosis of liver cancer and associated with poor survival.

\section{Data Availability}

The data used to support the findings of this study are included within the article.

\section{Conflicts of Interest}

The authors declare that there are no conflicts of interest regarding the publication of this paper.

\section{Acknowledgments}

To all of those who made this study possible, we express sincere gratitude. Special thanks to colleagues for writing excellent computer programs in $\mathrm{R}$, which supported us to collect and analyze a large amount of data.

\section{Supplementary Materials}

Figure legends: Supplementary Figure S1: the INPP5K mRNA expression in the GSE14520 cohort. The validation of INPP5K mRNA expression in liver cancer and normal liver tissues was conducted by GSE14520 cohort. Supplementary Figure S2: Kaplan-Meier curves for OS according to INPP5K expression in the ICGC cohort. The validation of survival analysis was conducted by ICGC cohort. (Supplementary Materials)

\section{References}

[1] R. Siegel, D. Naishadham, and A. Jemal, "Cancer statistics, 2013,” CA: A Cancer Journal for Clinicians, vol. 63, no. 1, pp. 11-30, 2013.

[2] T. Balla, "Phosphoinositides: tiny lipids with giant impact on cell regulation," Physiological Reviews, vol. 93, no. 3, pp. 10191137, 2013.

[3] M. J. Eramo and C. A. Mitchell, "Regulation of PtdIn$\mathrm{s}(3,4,5) \mathrm{P} 3 /$ Akt signalling by inositol polyphosphate 5-phosphatases," Biochemical Society Transactions, vol. 44, no. 1, pp. 240-252, 2016.

[4] T. Ijuin, Y. Mochizuki, K. Fukami, M. Funaki, T. Asano, and T. Takenawa, "Identification and characterization of a novel inositol polyphosphate 5-phosphatase," The Journal of Biological Chemistry, vol. 275, no. 15, pp. 10870-10875, 2000.

[5] D. P. S. Osborn, H. L. Pond, N. Mazaheri et al., "Mutations in INPP5K cause a form of congenital muscular dystrophy overlapping Marinesco-Sjögren syndrome and dystroglycanopathy," American Journal of Human Genetics, vol. 100, no. 3, pp. 537-545, 2017.

[6] T. Ijuin, Y. E. Yu, K. Mizutani et al., "Increased insulin action in SKIP heterozygous knockout mice," Molecular and Cellular Biology, vol. 28, no. 17, pp. 5184-5195, 2008.

[7] M. Wiessner, A. Roos, C. J. Munn et al., "Mutations in INPP5K, encoding a phosphoinositide 5-phosphatase, cause congenital muscular dystrophy with cataracts and mild cognitive impairment," American Journal of Human Genetics, vol. 100, no. 3, pp. 523-536, 2017.

[8] A. R. Ramos, W. Elong Edimo, and C. Erneux, "Phosphoinositide 5-phosphatase activities control cell motility in glioblastoma: two phosphoinositides $\mathrm{PI}(4,5) \mathrm{P} 2$ and $\mathrm{PI}(3,4) \mathrm{P} 2$ are involved," Advances in Biological Regulation, vol. 67, pp. 40-48, 2018.

[9] Team RDCJC, "R : a language and environment for statistical computing," R Foundation for Statistical Computing, Vienna, Austria, 2009.

[10] X. Robin, N. Turck, A. Hainard et al., "pROC: an open-source package for $\mathrm{R}$ and $\mathrm{S}+$ to analyze and compare ROC curves," BMC Bioinformatics, vol. 12, no. 1, 2011.

[11] T. M. Therneau, A package for survival analysis in S, 1994.

[12] T. M. Therneau and P. M. Grambsch, Modeling Survival Data: Extending the Cox Model, vol. 97, Springer, New York, 2000. 
[13] L. Hou, X. Zhang, Y. Jiao et al., "ATP binding cassette subfamily $\mathrm{B}$ member 9 (ABCB9) is a prognostic indicator of overall survival in ovarian cancer," Medicine (Baltimore), vol. 98, no. 19, article e15698, 2019.

[14] Y. Jiao, Z. Fu, Y. Li, L. Meng, and Y. Liu, "High EIF2B5 mRNA expression and its prognostic significance in liver cancer: a study based on the TCGA and GEO database," Cancer Management and Research, vol. Volume 10, pp. 6003-6014, 2018.

[15] Y. Jiao, Z. Fu, Y. Li, W. Zhang, and Y. Liu, "Aberrant FAM64A mRNA expression is an independent predictor of poor survival in pancreatic cancer," PLoS One, vol. 14, no. 1, article e0211291, 2019.

[16] Y. Jiao, Y. Li, P. Jiang, W. Han, and Y. Liu, "PGM5: a novel diagnostic and prognostic biomarker for liver cancer," PeerJ, vol. 7, article e7070, 2019.

[17] Y. Jiao, Y. Li, S. Liu, Q. Chen, and Y. Liu, "ITGA3 serves as a diagnostic and prognostic biomarker for pancreatic cancer," OncoTargets and Therapy, vol. Volume 12, pp. 4141-4152, 2019.

[18] Y. Jiao, Y. Li, Z. Lu, and Y. Liu, "High trophinin-associated protein expression is an independent predictor of poor survival in liver cancer," Digestive Diseases and Sciences, vol. 64, no. 1, pp. 137-143, 2019.

[19] Y. Li, Y. Jiao, Z. Fu, Z. Luo, J. Su, and Y. Li, "High miR-454-3p expression predicts poor prognosis in hepatocellular carcinoma," Cancer Management and Research, vol. 11, pp. 27952802, 2019.

[20] Y. Jiao, Y. Li, B. Jia et al., "The prognostic value of lncRNA SNHG4 and its potential mechanism in liver cancer," Bioscience Reports, vol. 40, no. 1, 2020.

[21] Y. Jiao, Y. Li, B. Ji, H. Cai, and Y. Liu, "Clinical value of IncRNA LUCAT1 expression in liver cancer and its potential pathways," Journal of Gastrointestinal and Liver Diseases, vol. 28, no. 4, pp. 439-447, 2019.

[22] Z. Fu, Y. Jiao, Y. Q. Li et al., "PES1 in liver cancer: a prognostic biomarker with tumorigenic roles," Cancer Management and Research, vol. 11, pp. 9641-9653, 2019.

[23] Y. C. Zhao, Y. Jiao, Y. Q. Li, Z. Fu, Z. Y. Yang, and M. He, "Elevated high mobility group A2 expression in liver cancer predicts poor patient survival," Revista Española de Enfermedades Digestivas, vol. 112, no. 1, pp. 27-33, 2020.

[24] Y. Jiao, Y. Li, P. Jiang, Z. Fu, and Y. Liu, "High MAST2 mRNA expression and its role in diagnosis and prognosis of liver cancer," Scientific Reports, vol. 9, no. 1, p. 19865, 2019.

[25] D. Yang, Y. Jiao, Y. Li, and X. Fang, "Clinical characteristics and prognostic value of MEX3A mRNA in liver cancer," Peer], vol. 8, article e8252, 2020.

[26] Y. Jiao, Y. Li, Z. Fu et al., "OGDHL expression as a prognostic biomarker for liver cancer patients," Disease Markers, vol. 2019, Article ID 9037131, 9 pages, 2019.

[27] N. Thapa, X. Tan, S. Choi, P. F. Lambert, A. C. Rapraeger, and R. A. Anderson, "The hidden conundrum of phosphoinositide signaling in cancer," Trends Cancer, vol. 2, no. 7, pp. 378-390, 2016.

[28] N. Thapa, Y. Sun, M. Schramp, S. Choi, K. Ling, and R. A. Anderson, "Phosphoinositide signaling regulates the exocyst complex and polarized integrin trafficking in directionally migrating cells," Developmental Cell, vol. 22, no. 1, pp. 116130, 2012.

[29] N. Thapa and R. A. Anderson, "PIP2 signaling, an integrator of cell polarity and vesicle trafficking in directionally migrating cells," Cell Adhesion \& Migration, vol. 6, no. 5, pp. 409-412, 2012.

[30] S. Choi, N. Thapa, A. C. Hedman, Z. Li, D. B. Sacks, and R. A. Anderson, "IQGAP1 is a novel phosphatidylinositol 4,5 bisphosphate effector in regulation of directional cell migration," The EMBO Journal, vol. 32, no. 19, pp. 2617-2630, 2013.

[31] W. S. E. Edimo, S. Ghosh, R. Derua et al., "SHIP2 controls plasma membrane $\mathrm{PI}(4,5) \mathrm{P} 2$ thereby participating in the control of cell migration in $1321 \mathrm{~N} 1$ glioblastoma cells," Journal of Cell Science, vol. 129, no. 6, pp. 1101-1114, 2016.

[32] A. R. Ramos, S. Ghosh, M. Dedobbeleer, P. A. Robe, B. Rogister, and C. Erneux, "Lipid phosphatases SKIP and SHIP2 regulate fibronectin-dependent cell migration in glioblastoma," The FEBS Journal, vol. 286, no. 6, pp. 1120-1135, 2019.

[33] J. Zucman-Rossi, A. Villanueva, J. C. Nault, and J. M. Llovet, "Genetic landscape and biomarkers of hepatocellular carcinoma," Gastroenterology, vol. 149, no. 5, pp. 1226-1239.e4, 2015.

[34] Y. Hoshida, A. Villanueva, M. Kobayashi et al., "Gene expression in fixed tissues and outcome in hepatocellular carcinoma," The New England Journal of Medicine, vol. 359, no. 19, pp. 1995-2004, 2008.

[35] Y. Hoshida, A. Villanueva, A. Sangiovanni et al., "Prognostic gene expression signature for patients with hepatitis Crelated early-stage cirrhosis," Gastroenterology, vol. 144, no. 5, pp. 1024-1030, 2013.

[36] L. Rimassa, E. Assenat, M. Peck-Radosavljevic et al., "Tivantinib for second-line treatment of MET-high, advanced hepatocellular carcinoma (METIV-HCC): a final analysis of a phase 3, randomised, placebo- controlled study," The Lancet Oncology, vol. 19, no. 5, pp. 682-693, 2018. 